\title{
Search for $W^{\prime}$ bosons through decays to boosted-top and boosted-bottom jets
}

\author{
Daniel Duffty* and Zack Sullivan ${ }^{*}$ \\ Department of Physics, Illinois Institute of Technology, Chicago, Illinois 60616-3793, USA
}

(Dated: July 6, 2013)

\begin{abstract}
We propose a novel model-independent method to search for $W^{\prime}$ bosons at the Large Hadron Collider by looking at dijets where one jet is identified as a boosted-top jet and another jet is tagged as a boosted-bottom jet. Performing a detector simulated signal and background study, we demonstrate that the reach in effective coupling $g^{\prime}$ is improved over existing analysis methods by up to factor of 2 for $W^{\prime}$ masses above $1.5 \mathrm{TeV}$, and the reach in mass is extended by $600 \mathrm{GeV}$ to $2.6 \mathrm{TeV}$ at an $8 \mathrm{TeV}$ Large Hadron Collider. We introduce a method for distinguishing high-energy $b$ jets from light-quark jets, and describe an important set of backgrounds to top-jets. We propose a series of data-driven samples that might be used to measure the efficiencies for these new backgrounds in the LHC data.
\end{abstract}

PACS numbers: 14.70.Pw,14.65.Ha,14.65.Fy,13.85.Rm

\section{INTRODUCTION}

Model-independent searches for new charged vector currents, generally called $W^{\prime}$ bosons, that decay to top and bottom quarks [1] are important tools for investigating physics beyond the standard model. Following our recent theoretical calculation of a model independent analysis at the Large Hadron Collider (LHC) [2], the CMS [3] and ATLAS [4] Collaborations set strong bounds on generic $W^{\prime}$ bosons as functions of effective coupling $g^{\prime}$. While tighter mass bounds exist for special cases, such as pure left-handed $W_{L}^{\prime}$ bosons using lepton transverse momentum spectra [5], the top quark final state exhibits a resonant mass peak can be fully reconstructed [1]. In this Letter we demonstrate the first use of boosted-top jets for improving the reach in both $W^{\prime}$ mass and effective coupling $g^{\prime}$. In addition, we introduce a new boostedbottom jet algorithm that provides the key to extending this analysis beyond existing measurements.

$W^{\prime}$ bosons appear in many classes of theories that stabilize electroweak symmetry breaking. High mass copies of $W$ boson resonances are proposed in some theories [6]; right-handed counterparts to the left-handed standard model $W$ are found in theories with a broken $S U(2)_{L} \times S U(2)_{R}$ symmetry [7-10]. Other theories, such as non-commuting extended technicolor [11], promote a heavy mass eigenstate. $W^{\prime}$ bosons enter the Lagrangian with terms of the form

$$
\mathcal{L}=\frac{g^{\prime}}{2 \sqrt{2}} V_{i j}^{\prime} W_{\mu}^{\prime} \bar{f}^{i} \gamma^{\mu}\left(1 \pm \gamma_{5}\right) f^{j}+\text { H.c. },
$$

which mirrors that of the standard model $W$ (without the lepton sector if it is right-handed). There could be left-right mixing, but such mixing is constrained by $K_{-}$ $\bar{K}$ mixing [12]. Theories have been proposed that would suppress this mixing via orbifold breaking of the left-right symmetry [13] or supersymmetric interactions [14].

In searches for $W^{\prime}$ boson resonances between $1-3 \mathrm{TeV}$, existing analyses [2-4] are limited by their ability to reconstruct isolated decay products of the top quark $(t \rightarrow b W \rightarrow b j j / b l \nu)$. As the mass of the $W^{\prime}$ boson increases, relativistic angle compression causes the top quark decay products to merge into a "fat jet," distinguishable from normal jets only by its substructure. Several "boosted top quark" tagging algorithms have been developed [15 17] to take advantage of these kinematics. So far these algorithms have shown promise in Higgs plus top quark searches [18] and $Z^{\prime} \rightarrow t \bar{t}$ events [19]. In this Letter, we examine the effectiveness of boosted-top tags in improving the reach to higher mass and smaller effective coupling.

In the first section of this Letter, we describe our simulation of the $W^{\prime}$ signal and backgrounds in a dijet sample with a top tag. We find naive theoretical estimates of boosted-top tag efficiencies and mistag rates are too optimistic, and adopt the efficiencies measured by the CMS Collaboration [16]. We also discuss a class of backgrounds to top tagging that have been previously ignored by experiments in hadronic channels, and place conservative upper limits on these backgrounds for the signal we consider.

We introduce a new "boosted bottom quark tag" in the second section of this Letter to improve the reach in mass and couplings. Utilizing the kinematic features of $\mathrm{TeV} b$ jets along with the muon decay channel of $B$ hadrons, we find reasonable tagging efficiencies and good fake rejection. Since our analysis is at the level of a fast detector simulation, we also recommend data samples where the efficiencies can be extracted.

Having described models for signals and backgrounds using the new boosted-top and bottom objects, we show that the reach in $W^{\prime}$ mass can be extended by $600 \mathrm{GeV}$ in the same amount of data as used in current limits. More importantly, we find that limits on the effective coupling $g^{\prime}$ can be improved by a factor of 2 over the previous analysis techniques for masses above $1.5 \mathrm{TeV}$. Hence, many perturbative models containing new charged-vector currents can be excluded up to $2.6 \mathrm{TeV}$. We conclude this 
Letter with suggestions for future research.

\section{BOOSTED-TOP TAGS AND BACKGROUNDS}

The process of interest is resonant $W^{\prime}$ production, with a decay into a boosted-top jet and a boosted-bottom jet $\left(p p \rightarrow W^{\prime} \rightarrow t b+X\right)$ at the LHC. The dominant backgrounds are expected to come from dijet production $(j j$, $c j, c \bar{c}, b j, b \bar{b}, b c)$, where showering creates "fat" jets that fake boosted-top jets, and standard model (SM) production of single top quarks in the $s$ - and $t$-channel $(t b, t j)$ and ditop $(t \bar{t})$, which pass boosted-top jet tags. In addition, we find a class of "accidental" backgrounds to boosted-top tags from $W j j$ and $Z j j$ that we examine below.

We simulate events by feeding the output of a general $W^{\prime}$ model [20] and backgrounds produced in MADEVENT 21] into PYTHIA 22] for showering and hadronization. We reconstruct events using an ATLAS-like detector model in a modified version of the PGS 4 23] detector simulation we have previously calibrated to ATLAS data 2]. Cross sections are scaled to next-to-leading order (NLO) using Ref. 2] for the $W^{\prime}$ signal, and MCFM 24] for backgrounds.

In order to make contact with experiment, we begin with a Cambridge-Aachen implementation of boostedtop tagging studied by the CMS Collaboration [16]. The CMS procedure is quite involved, but it roughly corresponds to finding a large $(\Delta R<0.8)$ hard jet whose mass loosely reconstructs to a top-quark mass between 100-250 GeV. In addition, the jet must decompose into three or four "subjets," of which a pair must combine to produce a $W$-like mass $(>50 \mathrm{GeV})$. Implementations of this top tagging algorithm are extremely sensitive to details of the detector simulation, such as non-uniform calorimeter segmentation. In implementing the CMS algorithm, we initially found top-jet acceptances $\sim 10 \%$ higher than observed by CMS, and fake rates from light jets nearly an order-of-magnitude lower than observed by CMS.

To reduce sensitivity to our detector model and provide a more realistic comparison between $W^{\prime}$ reconstruction using top jets and analyses where top decay products can be isolated 2], we fit transverse momentum $\left(p_{T}\right)$ dependent tagging efficiencies for top-jets and fakes rates provided by CMS in Figs. 13 and 14, respectively, of Ref. [16] and apply them to our reconstructed jets. ${ }^{1}$ While the CMS top-jet acceptance is asymptotically $45 \%$ at large

\footnotetext{
${ }^{1}$ Initial efficiency estimates by the ATLAS Collaboration using an anti- $k_{T}$ algorithm are quantitatively consistent with the fits we use here [25], suggesting low sensitivity to details of the algorithm.
}

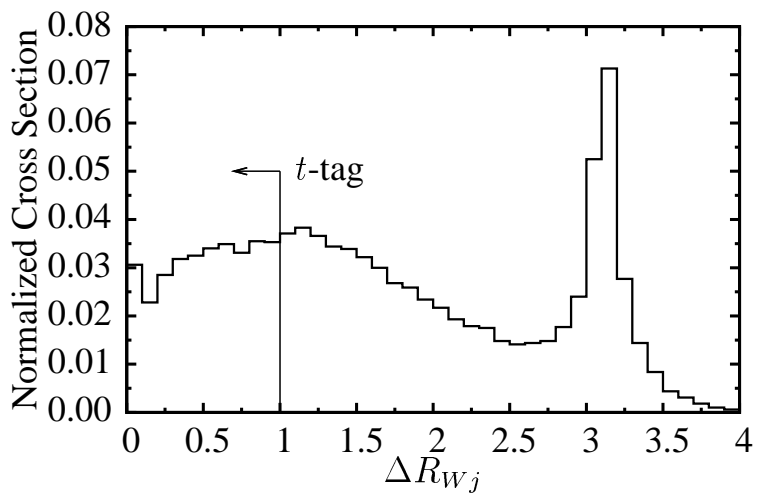

FIG. 1: $\Delta R$ between the $W$ and the nearest jet in $W j j$ events. Events with $\Delta R<1$ are candidates for being tagged as a top jet, provided they fit a loose top-mass cut.

$p_{T}$, our results are driven primarily by the fake rate, which ranges from $1-4 \%$ over the $p_{T}$ range we reconstruct. This large fake rate would cause $j j$ to completely dominate the signal if not for the new boosted-bottom tag introduced below.

Nothing in boosted-top jet algorithms requires the subjets to come from top quarks or showered light-quark jets (fakes). An important class of backgrounds to top tagging comes from $W j j / W b j$ or $Z j j$ events, where one jet fluctuates close enough to a hadronically decaying $W$ or $Z$ to pass the boosted-top algorithm. We model these contributions by using the detector simulation above to look for three jets in a cone of $\Delta R<1$ and applying a cut on the invariant mass of the three jets of $100<M_{j j j}<250 \mathrm{GeV}$. In Fig. 1 we see a significant fraction of $W j j$ events pass the cut in $\Delta R$, and we show below that this background is larger than either real standard model top or bottom quark processes after all cuts. Similar backgrounds in semi-leptonic boosted-top decays have been examined by considering muon proximity to jets [26]; and a parameter $z_{\mu}$ has been proposed [27] to suppress this non-top background. It is possible similar methods could be developed to suppress $W j+X$ contributions to hadronic boosted-top decays.

A significant challenge to estimating the backgrounds from $W j j$ or $Z j j$ comes from instabilities of the perturbative calculation in the highly restricted phase space when a jet is near the $W$. In order to place upper bounds on these contributions we estimate a theoretical error of $50 \%$ in the acceptance using two methods: First we look at increases in acceptance between leading order and NLO predicted by MCFM [24] due to higher order hard radiation near the $W$. In addition, we examine increases in acceptance between parton level and showering after PYTHIA and reconstruction. Both methods indicate at least $30 \%$ increases in boosted-top acceptance of $W j j$, $W b j$, and $Z j j$. Hence, we include an additional $K$-factor of 1.5 times the NLO cross section when calculating these backgrounds. Fortunately, these backgrounds are sup- 


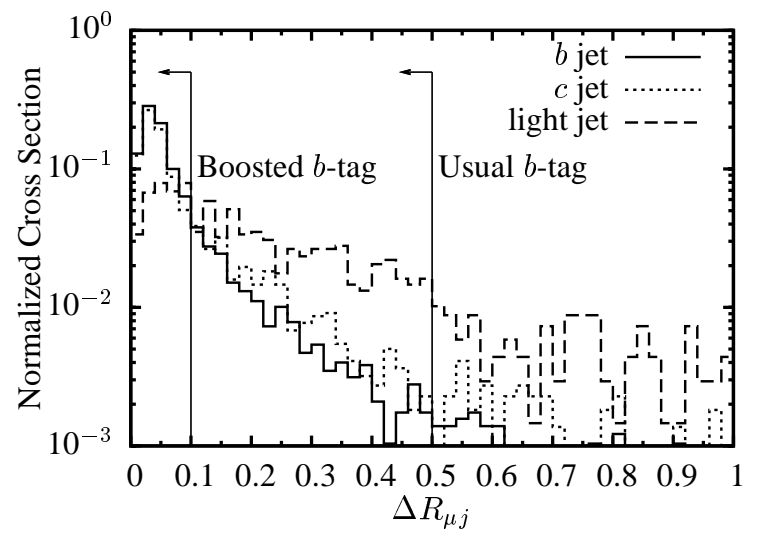

FIG. 2: $\Delta R$ separation between muons and the centroid of their associated jets in $b b, c c$, and $j j$ events for jets with $E_{T}>400 \mathrm{GeV}$. Muons from $b$ decays are more central than those from light quark jets.

pressed by other cuts, but future boosted-top studies should account for them.

\section{BOOSTED-BOTTOM JET TAGGING}

In a $W^{\prime} \rightarrow t b$ final state, the jet containing the $B$ hadron which recoils against the top jet is typically the highest energy jet in the event. Historically, the flavor content of the $b$-jet has been ignored [1, 3, 4, 28 31] because modern secondary vertex tagging is difficult above a few hundred $\mathrm{GeV}$. The decay products of $B$ hadrons with relativistic $\gamma=100-300$ are boosted to be approximately collinear, and hence tracking generally points back to the interaction vertex within the resolution of the detectors. In order to overcome the dijet backgrounds due to light-quark jets, it is necessary to identify these $b$ jets.

We propose a new "boosted-bottom tagging" algorithm that adds a kinematic twist to the old idea of $b$ tagging by observation of a muon inside of a jet. Beginning with data at the Fermilab Tevatron, muon-tagging of $b$ jets was relegated to enhancing the efficiency of secondary vertex tags. Typically, muons found within a 30 degree cone $\left(\Delta R_{\mu j} \sim 0.5\right)$ of the centroids of jets provide $10-15 \%$ of the $b$-tagging efficiency 23 ]. The fake rate for these muons is dominated by long-lived kaon and charged pion decays inside of the jets. As can be seen in Fig. 2 however, in jets with transverse energies of $E_{T}=500$ $1500 \mathrm{GeV}$ almost all of the muons from bottom or charm decays are boosted to within $\Delta R_{\mu j}<0.1$, while muons from light-quark or gluon initiated jets have a broader spectrum in $\Delta R_{\mu j}$. This contrasts with usual b-tagging, where some fraction of muons is either soft or at wide angles to the $b$ direction. Hence, our method for tagging boosted-bottom jets is to look for hits in a muon chamber within $\Delta R_{\mu j}<0.1$.
In addition to the smaller angle between the muon from $b$ decay and the jet, the transverse momentum of the muons from these boosted-bottom jets tends to be significantly harder that that of the light jets. We place a cut of $p_{T}>20 \mathrm{GeV}$ on the muon in order to aid reconstruction. This value corresponds to roughly the minimum possible muon energy in the LAB frame for these boosted kinematics. As luminosities increase at the LHC this threshold could be made proportional to the jet energy with a modest loss of acceptance. We extract transverse-energy $E_{T}$-dependent efficiencies for $b, c$, and light jet tags and list a few representative points in Tab. [ The ratio of $b$-tagging efficiency to fake rates is similar to what is currently achieved by secondary vertex tagging. The drop in efficiency below $400 \mathrm{GeV}$ is an artifact of using a fixed angular cut to maintain $b$ jet purity.

TABLE I: Boosted-bottom jet efficiencies using a muon tag with $p_{T \mu}>20 \mathrm{GeV}$ and $\Delta R_{\mu j}<0.1$ for $b$ jets, $c$ jets, and light jets $j$ as a function of jet $E_{T}$.

\begin{tabular}{cccc}
\hline \hline Type & $E_{T j}=100 \mathrm{GeV}$ & $400 \mathrm{GeV}$ & $1000 \mathrm{GeV}$ \\
\hline$b$ & $4.8 \%$ & $11.8 \%$ & $15.0 \%$ \\
$c$ & $2.1 \%$ & $5.5 \%$ & $7.5 \%$ \\
$j$ & $0.1 \%$ & $0.4 \%$ & $0.6 \%$ \\
\hline \hline
\end{tabular}

For this study we use boosted-bottom tag and fake rates estimated via Monte Carlo, but it is important to confirm these rates in data. Ideally one would want to examine a $b \bar{b}$ final state where the energies of the $b$ jets are near to the final boosted energies, however secondary vertexing is limited near $1 \mathrm{TeV}$. One promising sample would be $b b j$, where one boosted- $b$ jet recoils against a softer $b$ jet that is secondary vertex- (or muon-) tagged, and a light-quark or gluon jet. Based on our analyses there may be a few hundred boosted- $b$ jets near $1 \mathrm{TeV}$ in the $20 \mathrm{fb}^{-1}$ sample already collected at the LHC. The fake rate could be obtained by comparing to the much larger high- $E_{T}$ dijet sample. Another possible sample with some reach for boosted $b$-tags may be the high- $E_{T}$ tail of the dilepton channel of $t \bar{t}$ production, where the boosted- $b$ jet contains a muon, and the second lepton is isolated enough to be distinguished. Given the size of the background from $t \bar{t}$ to the standard model-independent analysis, there may be enough isolated events to get initial estimates of the efficiencies.

\section{$W^{\prime}$ SEARCH WITH BOOSTED- $t$ AND $b$ JETS}

We now turn to using boosted-top jet tags and boosted-bottom jet tags to examine their reach in probing for $p p \rightarrow W^{\prime} \rightarrow t b$ in the dijet sample for masses above $1 \mathrm{TeV}$. Unlike the analysis of Ref. [2], angular correlations of the top decay products are hidden by the 
kinematics of the two body decay. While interference between left-handed $W_{L}^{\prime}$ bosons with the standard model affects the total production cross section, we reconstruct events in the resonance region where the interference is negligible. Hence, we are left with tagging as one of the few handles in the analysis.

We begin with a sample of reconstructed central high energy dijets with $E_{T j_{1}}>0.38 M_{W^{\prime}}, E_{T j_{2}}>0.1 M_{W^{\prime}}$, $\left|\eta_{j}\right|<1.5$, and $\Delta R_{j j}>0.4$. We demand the pseudorapidity between the two jets is also small $\left|\eta_{j_{1}}-\eta_{j_{2}}\right|<1.5$ in order to suppress large angle contributions to the dijet invariant mass $M_{j j}$. For each $W^{\prime}$ mass we reconstruct a dijet invariant mass in $0.95 M_{W^{\prime}}<M_{j j}<1.05 M_{W^{\prime}}$. We set $95 \%$ confidence level (C.L.) exclusion limits after cuts.

In Tab. II we list the number of signal $S$ and background $B$ events expected for a $2 \mathrm{TeV} W^{\prime}$ with $g^{\prime} / g_{S M}=$ 1 and $20 \mathrm{fb}^{-1}$ at a $8 \mathrm{TeV}$ LHC. After acceptance cuts, direct dijet production in QCD is 1000 times the signal. Adding a boosted-top tag improves $S / B$ to $1 / 33$, but the accidental backgrounds $W j j / W b j$ and $Z j j$ are larger than standard model $t \bar{t}$, single top, and comparable or larger than heavy-flavor dijets. Hence, future analyses of boosted-top jets should not ignore these accidentals.

TABLE II: Number of $W^{\prime} \rightarrow t b$ signal and background events predicted for a $2 \mathrm{TeV} W^{\prime}$ boson in $20 \mathrm{fb}^{-1}$ of integrated luminosity at an $8 \mathrm{TeV}$ LHC. Events are listed after acceptance cuts, with a boosted-top tag ( $+t$-tag), and adding a boostedbottom tag (+b-tag). Accidental backgrounds $W j j / W b j$ and $Z j j$ are not present before top tagging.

\begin{tabular}{lrrr}
\hline \hline Events & After acceptance & $+t$-tag & $+b$-tag \\
\hline$W^{\prime}$ signal & 702 & 316 & 47 \\
$j j$ & 735000 & 11000 & 66 \\
$c \bar{c}, c j$ & 19300 & 289 & 12 \\
$b \bar{b}, b j, b c$ & 10800 & 162 & 14 \\
$t \bar{t}, t j, t b$ & 51 & 25 & 4 \\
$W b j, W j j, Z j j$ & $*$ & 93 & 1 \\
\hline \hline
\end{tabular}

In Figs. 3 and 4 we see that using a boosted-top tag improves the $95 \%$ C.L. exclusion limit on $g^{\prime} / g_{S M}$ slightly for $M_{W^{\prime}}>2 \mathrm{TeV}$ if $g^{\prime} / g_{S M}>1$. The region $g^{\prime} / g_{S M}>1$ is difficult to measure as the width of the $W^{\prime}$ resonance grows rapidly with coupling $\left(\Gamma \sim 2.5 \% \times M_{W^{\prime}}\left(g^{\prime} / g_{S M}\right)^{2}\right)$. We treat the full width effects using Refs. [2, 20] since naive coupling scaling [1, 2] is only valid for $g^{\prime} / g_{S M}<1$. Small differences between right- and left-handed $W^{\prime}$ are due to different $t b$ branching fractions. In Fig. 4 we show both positive or negative interference between $W_{L}^{\prime}$ and the standard model. As expected, the interference is tiny in the resonance region.

In order to reach the full potential of this channel we next apply the new boosted-bottom tag. We see in Tab. III the sample purity $S / B$ improves to $1 / 2$, and the sig-

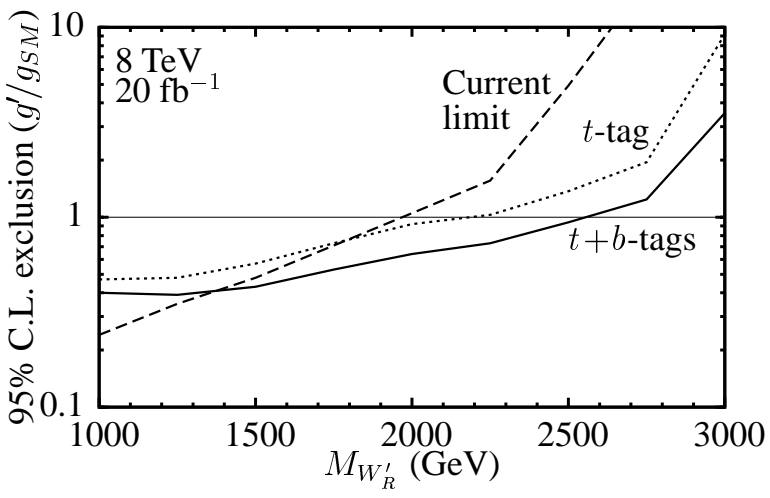

FIG. 3: 95\% C.L. limit on the effective coupling $g_{R}^{\prime}$ relative to $g_{S M}$ as a function of right-handed $W_{R}^{\prime}$ mass. Curves show the reach from current resolved-top quark analyses (dashed), the boosted-top analysis (dotted), and after adding a boostedbottom tag (solid).

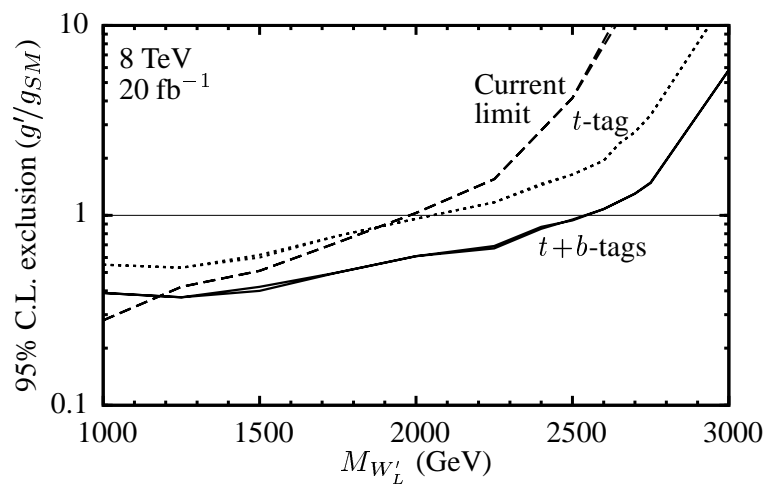

FIG. 4: 95\% C.L. limit on the effective coupling $g_{L}^{\prime}$ relative to $g_{S M}$ as a function of left-handed $W_{L}^{\prime}$ mass. Constructive (lower lines) and destructive (higher lines) interference with the standard model are nearly indistinguishable.

nificance $S / \sqrt{B}$ improves by $50 \%$ for $M_{W^{\prime}}=2 \mathrm{TeV}$. The boosted-bottom tag increases the reach in $W^{\prime}$ mass with $g^{\prime} / g_{S M}=1$ by $600 \mathrm{GeV}$. More importantly, the limit in $g^{\prime} / g_{S M}$ improves by nearly a factor of 2 over existing approaches for masses above $1.5 \mathrm{TeV}$, and remains perturbative to $3 \mathrm{TeV}$. Hence, models with slightly greater than standard model coupling, like orbifolded left-right breaking [13], can be ruled out for masses below $3 \mathrm{TeV}$. Combined with previous analyses below $1.5 \mathrm{TeV}$, a wide range of perturbative models can be excluded for masses below $2.6 \mathrm{TeV}$.

\section{CONCLUSIONS}

In this Letter we present the first use of boosted-top tags and introduce boosted-bottom tags in the search for new charged vector currents, called $W^{\prime}$ bosons, by looking at the $t b$ dijet final state. We present a quantitative estimate of "accidental" backgrounds to boosted-top jet 
measurements which are comparable to or larger than all backgrounds other than direct dijet $j j$. We implement a detector simulated analysis whose efficiencies are fit to data, and find that boosted-top jets provide modest improvement on existing analyses of the $t b$ final state which use isolated leptons and jets from top-quark decay.

The key to improving the experimental reach in $W^{\prime}$ mass by $600 \mathrm{GeV}$, and model-independent coupling $g^{\prime}$ by roughly a factor of 2 , is the use of a new "boostedbottom jet tag." This boosted-bottom tag should be useful beyond the signal presented here. In particular, studies of $Z^{\prime}$ production to boosted-top can be supplemented by studies of $Z^{\prime} \rightarrow b \bar{b}$. The smaller acceptance may be compensated by the greater background rejection from boosted-bottom tagging, and enhanced coupling to down-type quarks than to up-type quarks. Any channel with bottom jets in the final state is now open to direct observation.

Note added: After submission of this Letter, studies of pileup at a $14 \mathrm{TeV}$ high luminosity LHC suggest boosted top tags will remain efficient [32].

This work is supported by the U.S. Department of Energy under Contract No. de-sc0008347.

* Electronic address: dduffty@IIT.edu

† Electronic address: Zack.Sullivan@IIT.edu

[1] Zack Sullivan, Phys. Rev. D 66, 075011 (2002).

[2] Daniel Duffty and Zack Sullivan, Phys. Rev. D 86, 075018 (2012).

[3] S. Chatrchyan et al. (CMS Collaboration), Phys. Lett. B 718, 1229 (2013).

[4] G. Aad et al. (ATLAS Collaboration), Phys. Rev. Lett. 109, 081801 (2012).

[5] CMS Collaboration, CMS PAS EXO-12-060.

[6] A. Datta, P. J. O'Donnell, Z. H. Lin, X. Zhang and T. Huang, Phys. Lett. B 483, 203 (2000).

[7] J. C. Pati and A. Salam, Phys. Rev. D 10, 275 (1974).

[8] R. N. Mohapatra and J. C. Pati, Phys. Rev. D 11, 566 (1975).
[9] R. N. Mohapatra and J. C. Pati, Phys. Rev. D 11, 2558 (1975).

[10] G. Senjanovic and R. N. Mohapatra, Phys. Rev. D 12, 1502 (1975).

[11] R. S. Chivukula, E. H. Simmons and J. Terning, Phys. Rev. D 53, 5258 (1996).

[12] D. E. Groom et al. (Particle Data Group Collaboration), Eur. Phys. J. C 15, 1 (2000).

[13] Y. Mimura and S. Nandi, Phys. Lett. B 538, 406 (2002).

[14] M. Cvetic and J. C. Pati, Phys. Lett. B 135, 57 (1984).

[15] D. E. Kaplan, K. Rehermann, M. D. Schwartz, and B. Tweedie, Phys. Rev. Lett. 101, 142001 (2008).

[16] CMS Collaboration, CMS-PAS-JME-09-001.

[17] L. G. Almeida, S. J. Lee, G. Perez, I. Sung, and J. Virzi, Phys. Rev. D 79, 074012 (2009).

[18] T. Plehn, G. P. Salam, and M. Spannowsky, Phys. Rev. Lett. 104, 111801 (2010).

[19] G. Aad et al. (ATLAS Collaboration), J. High Energy Phys. 1301, 116 (2013).

[20] Yaofu Zhou and Zack Sullivan, "Modeling $W^{\prime}$ bosons for use with model independent studies," paper in production.

[21] J. Alwall et al., J. High Energy Phys. 0709, 028 (2007).

[22] T. Sjostrand, S. Mrenna, and P. Z. Skands, J. High Energy Phys. 0605, 026 (2006).

[23] J. Conway et al., http://www.physics .ucdavis.edu/ conway/resea]

[24] J. M. Campbell and R. K. Ellis, Nucl. Phys. Proc. Suppl. 205-206, 10 (2010).

[25] J. Love, private communication.

[26] K. Rehermann and B. Tweedie, J. High Energy Phys. 1103, 059 (2011).

[27] J. Thaler and L. -T. Wang, J. High Energy Phys. 0807, 092 (2008).

[28] D. Acosta et al. (CDF Collaboration), Phys. Rev. Lett. 90, 081802 (2003).

[29] T. Aaltonen et al. (CDF Collaboration), Phys. Rev. Lett. 103, 041801 (2009).

[30] V. M. Abazov et al. (D0 Collaboration), Phys. Lett. B 641, 423 (2006).

[31] V. M. Abazov et al. (D0 Collaboration), Phys. Lett. B 699, 145 (2011).

[32] R. Calkins et al., in "Proceedings of the Community Summer Study 2013 - Snowmass on the Mississippi," SNOW13-00075, arXiv:1307.6908 [hep-ph]. 